\title{
On the Nanoaggregated Emitter of All sp²-Hybridized Bistriphenylenyl in the Device Layout of Organic Light-Emitting Diodes
}

\author{
Jian-Yuan Yu, ${ }^{\dagger}$ I-Wen Peter Chen, ${ }^{\dagger}$ Chun-hsien Chen, ${ }^{*},+$ Sheng-Jui Lee,${ }^{\dagger}$ I-Chia Chen, $*, \dagger$ \\ Chang-Sheng Lin, ${ }^{\dagger}$ and Chien-Hong Cheng*,,
}

Department of Chemistry, National Tsing Hua University, Hsinchu, Taiwan, 30013, and Department of Chemistry, National Taiwan University, Taipei, Taiwan 10617

Received: July 28, 2007; In Final Form: November 7, 2007

\begin{abstract}
Atomic force microscopy (AFM) is employed to unveil the film morphology of 2,2'-bistriphenylenyl (BTP), an all $\mathrm{sp}^{2}$-hybridized polyaromatic blue emitter derived from two triphenylenes (TP) in the OLED layout (organic light-emitting diode). It is generally believed that molecules with such planar cores require derivatization or steric hindrance to hamper intermolecular $\pi-\pi$ stacking and reduce self-absorption. X-ray crystallographic results of BTP crystals show that the two triphenylenyls are coplanar, indicative of a likely tendency toward self-quenching. Surprisingly, AFM reveals grainy nanoaggregates of the BTP layer in the device, yet BTP films exhibit excellent performance for OLED applications. To correlate luminescent spectra with the molecular structure, BTP films of crystalline, nanoaggregated, and amorphous features are prepared and subjected to spectroscopic studies. These AFM and spectroscopic results in conjunction with simulation results of a small torsion barrier to rotate the TP rings suggest that in the OLED device BTP molecules adopt a range of dihedral angles which confer nonself-quenched BTP even without being derivatized with steric side chains.
\end{abstract}

\section{Introduction}

Stimulated by the booming demands of global energy conservation and innovative products such as organic lightemitting diodes (OLEDs), ${ }^{1-3}$ organic thin-film transistors (OTFTs $),{ }^{4,5}$ liquid crystal displays,${ }^{6}$ and solar cells, ${ }^{7-13}$ enormous research efforts have focused on the design and synthesis of polyarylenes with core moieties of planarity, rigidity, and $\pi$-conjugation. Their optoelectronic properties, for example, highest occupied molecular orbital-lowest unoccupied molecular orbital (HOMO-LUMO) energy gaps or the absorptionemission wavelengths, can conceptually be tuned by rationally tailored structures and by derivatized functionality. However, the conjugated planarity has a tendency of developing small crystallites $^{14,15}$ in thin films due to the intermolecular $\pi-\pi$ interactions. For OLEDs, the spontaneous crystallization can result in nonradiative self-absorption ${ }^{16}$ and impairs the performance of the devices. The domain boundaries between the crystallites trap electrons, retard the charge mobility, and thus elevate the operating voltage. ${ }^{16-18}$ Further deterioration in performance may take place when more crystalline domains and interstices are developed in the film. ${ }^{19}$ Therefore, amorphous films are favorable for optoelectronic applications.

To obstruct the tendency of $\pi-\pi$ stacking between the aromatic cores, alkyl derivatives ${ }^{20-23}$ or other steric hindrance are integrated to intervene the planarity. Typical examples include starburst, ${ }^{24-26}$ bulky aromatics, ${ }^{27-31}$ tetrahedral, ${ }^{32-34}$ spiro, ${ }^{35-38}$ and binaphthyl ${ }^{39-41}$ structures. The starburst-shape molecules are mostly triarylamine-based dendritics and are

* To whom correspondence should be addressed. E-mail: (C.-h.C.) chhchen@ntu.edu.tw; (I-C.C.)icchen@mx.nthu.edu.tw; (C.-H.C.) chcheng@mx.nthu.edu.tw.

$\dagger$ National Tsing Hua University.

$\doteqdot$ National Taiwan University. generally employed as hole-transporting layers in OLEDs. The nonplanarity arises from the pyramidal nitrogen centers. ${ }^{24-26}$ Phenyl, ${ }^{27-30}$ pryenyl, ${ }^{30,31}$ or other bulky aromatics attached as the sidegroups or endgroups can introduce steric hindrance. Tetrahedral-type structures are synthesized by introducing an $\mathrm{sp}^{3}$-hybridized carbon core ${ }^{32-34}$ in between a biphenyl and two other aryls. Similarly for spiro-based compounds, two fluorenes are fused by an $\mathrm{sp}^{3}$-hybridized carbon center such that one fluorene is perpendicular to the other. In the case of binaphthyls, alkoxyls are derivatized at the $2,2^{\prime}$ positions and render a dihedral angle of $60 \sim 120^{\circ}$ between the two naphthyls. ${ }^{42}$ In these examples, amorphous films are promoted by steric derivatives or by a center atom that adopts $\mathrm{sp}^{3}$ orbitals and breaks the molecular planarity.

Herein we investigate 2,2'-bistriphenylenyl (BTP), a polyaromatic blue emitter whose carbon atoms are all $\mathrm{sp}^{2}$-hybridized. Optical micrographs of BTP powders purified by sublimation (Figure 1) show distinct facets, suggesting that crystallization of BTP takes place facilely. X-ray crystallographic results reveal a herringbone packing in which the two triphenylenyls are coplanar. Without derivation or further synthetic decoration to the planar cores, BTP was expected to exhibit low EL (electroluminescence) efficiency due to its crystallinity and selfquenching. Surprisingly, BTP functions excellently as an emitting layer. We previously fabricated OLEDs of ITO/NPB$(40 \mathrm{~nm}) / \mathrm{BTP}(20 \mathrm{~nm}) / \mathrm{TPBI}(40 \mathrm{~nm}) / \mathrm{Mg}: \mathrm{Ag} / \mathrm{Ag}$ that exhibit external quantum efficiency, current efficiency, power efficiency, and brightness of $2.8 \%, 2.7 \mathrm{~cd} / \mathrm{A}, 0.63 \mathrm{~lm} / \mathrm{W}$, and $14954 \mathrm{~cd} / \mathrm{m}^{2}$, respectively. ${ }^{43}$ Such performance suggests that self-absorption is not a dominant pathway for energy dissipation. To the contrary, the EL peak at $447 \mathrm{~nm}$ (Figure 2, dashed trace $),{ }^{43}$ equivalent to a $78 \mathrm{~nm}(0.58 \mathrm{eV})$ red shift from the PL (photoluminescence) of BTP dissolved in toluene (Figure 2, dotted trace), indicates formation of aggregates or intermolecular 

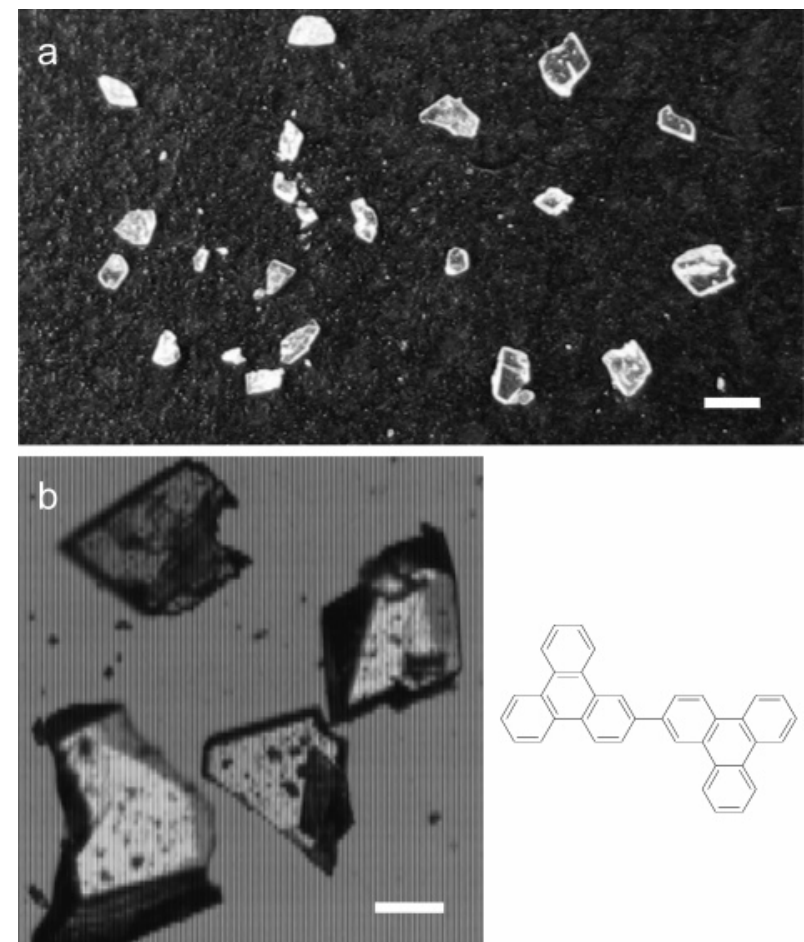

Figure 1. Chemical structure of BTP and optical micrographs of the sublimation-purified powders. Scale bars: (a) $0.5 \mathrm{~mm}$, (b) $50 \mu \mathrm{m}$.

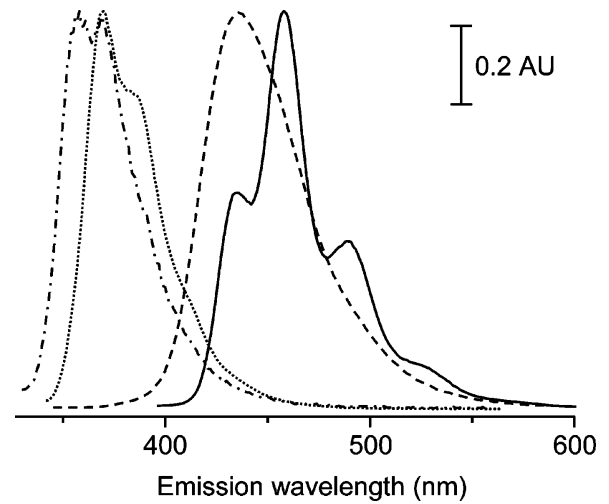

Figure 2. Normalized luminescent spectra of TP in toluene (dashdotted trace, $25 \mu \mathrm{M}$ ), BTP in toluene (dotted, $6 \mu \mathrm{M}$ ), BTP powders on glass slide (solid), and BTP in an OLED device (dashed). All the spectra are PL except the one from the OLED device (see text) whose EL instead of PL is displayed because the hole and electron transporting layers emit PL as well.

interactions. To rationalize the contradictory results, the morphology of BTP films are studied in the present study by tapping-mode atomic force microscopy (TM-AFM), which exerts minimal force and preserves the film structure. ${ }^{44-46}$ Because the intriguing EL performance of BTP is developed from nanometer-thick films in OLED devices, resolving the morphological feature of the BTP layer on closely resembled substrate will facilitate apparent correlation between the thin film structure and their molecular electronic states.

\section{Experimental Section}

Materials. Synthesis of BTP was documented in detail elsewhere. ${ }^{43,47}$ Substrates of HOPG (highly oriented pyrolytic graphite), silicon wafer, and ITO (indium-tin oxide) were obtained, respectively, from Structure Probe Incorporation (434HP-AB, SPI, West Chester, PA), Phoenix Silicon International (Psi Corp., Hsinchu, Taiwan), and Merck. All chemicals were reagent grade.
Preparation of OLED Devices and BTP Films. For EL measurements, the BTP-based OLEDs were fabricated by thermal evaporation of the materials of hole transporting layer (HTL), emitting layer, and electron transporting layer (ETL) successively onto an ITO electrode below $5 \times 10^{-6}$ Torr. The deposition rate for the organic compounds was $1 \AA / \mathrm{s}$. The cathode was then made by coevaporation of $\mathrm{Mg} / \mathrm{Ag}$ alloy (10: $1,100 \mathrm{~nm}$ ) followed by vacuum deposition of $\mathrm{Ag}$. The co-deposition rates for $\mathrm{Ag}$ and $\mathrm{Mg}$ were 1 and $10 \AA / \mathrm{s}$, respectively, and the deposition rate for $\mathrm{Ag}$ was $3.5 \AA / \mathrm{s}$. For morphological studies by AFM, BTP thin films were grown on HOPG, Si-wafer, and ITO by vacuum deposition $\left(<5 \times 10^{-6}\right.$ Torr) with a deposition rate of $1 \AA / \mathrm{s}$. The deposition rates and film thickness were determined by a quartz crystal microbalance equipped in the thermal evaporator (Auto 168, Junsun Tech Co., Taipei, Taiwan).

PL and EL Measurements. The spectra were recorded on a Hitachi F-4500 fluorescence spectrophotometer at room temperature. One centimeter quartz cuvets were used for solution samples. For solid samples or films, the light was incident at $\sim 15^{\circ}$ with respect to the surface normal.

AFM Measurements. TM-AFM measurements were carried out with a NanoScope IIIa controller (Veeco Metrology Group/ Digital Instruments, Santa Barbara, CA). Images were acquired using a $10 \mu \mathrm{m}$ scanner and monolithic silicon cantilevers (NSC15/AlBS, MikroMash, Madrid, Spain) whose force constant and the typical tip curvature were $40 \mathrm{nN} / \mathrm{m}$ and $10 \mathrm{~nm}$, respectively. The microscope was housed in a Plexiglas chamber through which dry $\mathrm{N}_{2}$ was purged throughout the experiments, and the humidity was kept lower than $2 \%$.

Computation Detail. Theoretical calculations were performed with the GAUSSIAN 03 program by using HP Superdome workstation. The density functional theory (DFT) was used with method B3LYP (the hybrid three parameter Becke exchange functional ${ }^{48}$ combined with the Lee-Yang-Parr correlation functional ${ }^{49}$ ) and basis set $6-31 G^{*}$ to obtain the optimized geometries of the ground states and various conformation. Harmonic vibrational frequencies were calculated with the optimized geometries and were used to obtain the zeropoint energies and the optimized geometries by the number of imaginary frequencies.

\section{Results and Discussions}

Electroluminescence and Photoluminescence Spectra. To explore the conformation of BTP in OLEDs, a typical luminescence spectrum of a BTP-based device is compared with those of BTP in toluene $(6 \mu \mathrm{M})$ and those being the powder form that represent molecular structures, correspondingly, with large and small dihedral angles between the triphenylenyl rings (vide infra). The OLED device that exhibits the spectrum in Figure 2 (dashed line) consists of layers of ITO/NPB(40 nm)/ $\mathrm{BTP}(20 \mathrm{~nm}) / \mathrm{TPBI}(30 \mathrm{~nm}) / \mathrm{Mg}: \mathrm{Ag}$ where ITO, NPB, and TPBI are the anode, the hole transporting layer, and the electron transporting layer, respectively, abbreviated for indium-tin-oxide, $N, N^{\prime}$-bis-(1-naphthyl)- $N, N^{\prime}$-diphenyl-1,1'-biphenyl-4,4'-diamine, and 1,3,5-tris(phenyl-2-benzimidazoly)benzene. While PL will be used to correlate the molecular structures, presented in Figure 2 for the BTP film in the device is an EL spectrum because the layers of NPB and TPBI also exhibit PL, which makes difficult the interpretation of BTP emission. The device produces an EL emission band with a maximum $\left(\lambda_{\max }\right)$ at 447 $\mathrm{nm}$, an FWHM of ca. $65 \mathrm{~nm}$, and an intense blue light (14 945 $\mathrm{cd} / \mathrm{m}^{2}$ at $\left.14 \mathrm{~V}\right)$ with a CIE coordinate of $(0.15,0.06)$.

Upon irradiation with 349-nm light, BTP in toluene shows a $\lambda_{\max }$ at $369 \mathrm{~nm}$ (the dotted line in Figure 2). Also displayed is 

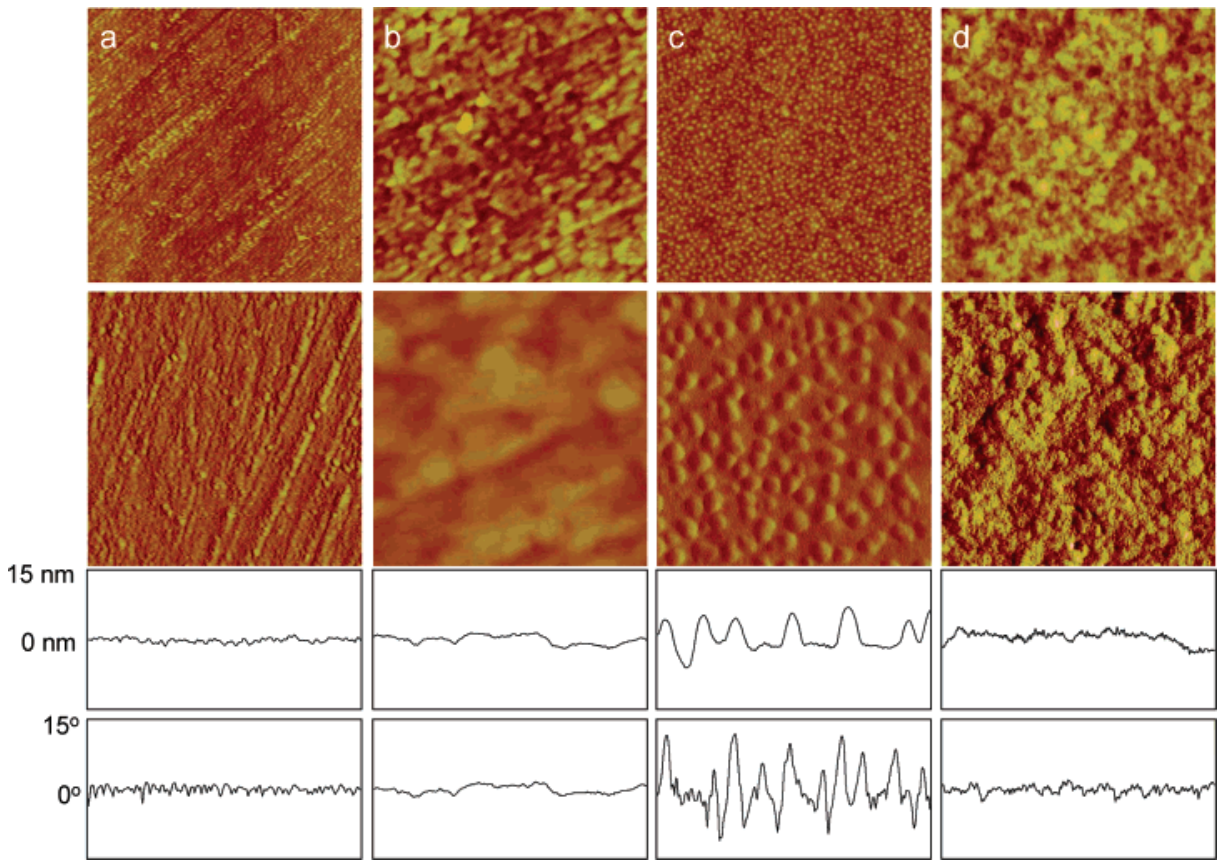

Figure 3. Typical TM-AFM images of height mode (upper row, in $3.8 \mu \mathrm{m}$ scan size), phase mode (middle row, in $1.00 \mu \mathrm{m}$ scan size, obtained from different samples to show the generality), cutaway view of height modes (third row, in $1.00 \mu \mathrm{m}$ scan size), and phase modes (bottom row, in $1.00 \mu \mathrm{m}$ scan size) for (a) ITO substrate, (b) ITO/NPB(40 nm), (c) ITO/NPB(40 nm)/BTP(1 nm), and (d) ITO/NPB(40 nm)/BTP(20 nm). The values in the parenthesis indicate the film thickness monitored by a quartz crystal resonator during thermal evaporation. Vertical scales for the image: $10 \mathrm{~nm}$ and $5^{\circ}$ for panels a, b, and d; $30 \mathrm{~nm}$ and $40^{\circ}$ for panel c. The drive frequency of the tip: $333 \mathrm{~Hz}$. Line scan rate for 512 lines per frame: $1.0 \mathrm{~Hz}$.

a PL spectrum of triphenylene (TP) whose structure is essentially a bisected BTP. The PL shows a peak located at $358 \mathrm{~nm}$, which is only $11 \mathrm{~nm}$ shorter than that of BTP. This difference is smaller than the 35 50 nm red-shift found when conjugated polyenes gain an additional double bond, ${ }^{50}$ suggesting a BTP conformation having two noncoplanar TPs.

The solid trace in Figure 2 is obtained from BTP powders and has three distinct emission peaks at 434, 458, $492 \mathrm{~nm}$ and a shoulder at ca. $527 \mathrm{~nm}$. The characteristics of multiple bands have been previously ascribed to vibronic features or conformational isomers. ${ }^{43}$ Our recent crystallographic results reveal that TP moieties of BTP are coplanar, and the molecules adopt a herringbone packing motif. The coplanarity extends the conjugation length and may account for the $\sim 90 \mathrm{~nm}$ red-shift of the PL bands from the solution phase to crystallinity. Similarly, the $\lambda_{\max }$ of the EL band is red-shifted by $\sim 80 \mathrm{~nm}$ from that of the solution BTP and is adjacent to the peak positions of BTP powders. BTP in the device may have a nearly coplanar conformation for the two TP moieties. Because intermolecular $\pi-\pi$ stacking developed in solids can cause red shift of $\lambda_{\max },{ }^{51-53}$ the luminescent spectra suggest that packing structures of BTP in the film and in powders are analogous to some extent.

Morphological Feature of BTP Films. Figure 3 shows typical TM-AFM images of bare ITO, ITO/NPB(40 nm), ITO/ $\mathrm{NPB}(40 \mathrm{~nm}) / \mathrm{BTP}(1 \mathrm{~nm})$, and ITO/NPB(40 nm)/BTP(20 nm). These samples represent the chronicle order of layer-by-layer fabrication where the third sample with the $1 \mathrm{~nm}$ thick BTP layer mimics the initial stage of the $20 \mathrm{~nm}$ BTP layer. ITO substrates are microscopically flat and show polishing scratches (Figure 3a) that are still discernible after depositing a layer of $40 \mathrm{~nm}$ thick NPB. For example, Figure $3 \mathrm{~b}$ shows the polishing marks going from upper right to lower left even though the topography is composed of submicron-sized domains.

TM-AFM is powerful in characterizing composite materials. Acquired simultaneously with the height mode, phase images measure the difference in phase angle of the actual tip oscillation from that of the driving piezoelectric oscillator. The phase shift is sensitive to different tip-sample interactions and thus reflects the distribution of deposited molecules on the substrates. In the case of Figure $3 \mathrm{~b}$, the phase image shows granular boundaries of NPB domains. The nominal phase shift and the maximum variation in height along the section profile are within $2^{\circ}$ and $2.5 \mathrm{~nm}$, respectively. The visible underlying scratches and the featureless phase-mode images of ITO/NPB(40 nm) suggest a uniform NPB overlayer on the ITO electrode.

Thermally evaporating 1-nm thick BTP on ITO/NPB (40 nm) results in circular aggregates (Figure $3 \mathrm{c}$ ) that are nominally 56 $\pm 10 \mathrm{~nm}$ in diameter and appear $10.8 \pm 1.5 \mathrm{~nm}$ higher than the underlying NPB. The most striking finding is manifested by the section profile of the phase image. The peak-to-peak phase shift reaches $17^{\circ}$, evidently due to inhomogeneous distribution (namely, nanoaggregates) of the BTP molecules. After further deposition of 20-nm BTP, the films still exhibit features of circular domains (Figure 3d). The grains are no longer protrusion-like, and the phase shift is reduced to $<4^{\circ}$, suggesting that the evaporated BTP molecules fill in the interstices among the grainy aggregates. Interestingly, the averaged diameter increases only slightly to $62 \pm 8 \mathrm{~nm}$, and the density of grains increases from 42 to 104 per $\mu \mathrm{m}^{2}$. The fact that BTP molecules tend to preserve the size of aggregates supports the possibility that BTP upholds crystallinity to some extent.

Correlation between Film Morphology and Photophysics. To further examine the effect of BTP packing on photoluminescence, a comparison is made for PL spectra measured from three distinct morphologies. Microscopic structures of $2.0 \mathrm{~nm}$ thick BTP films are studied by TM-AFM, and the typical images are displayed in Figure 4. The thickness is gauged by a quartz crystal microbalance (QCM) resonator during thermal evaporation. BTP films on graphite and on untreated $\mathrm{Si}$ wafers are discontinuous and therefore, exhibit aggregates taller than the deposited thickness of $2.0 \mathrm{~nm}$. The section profiles at the lower 

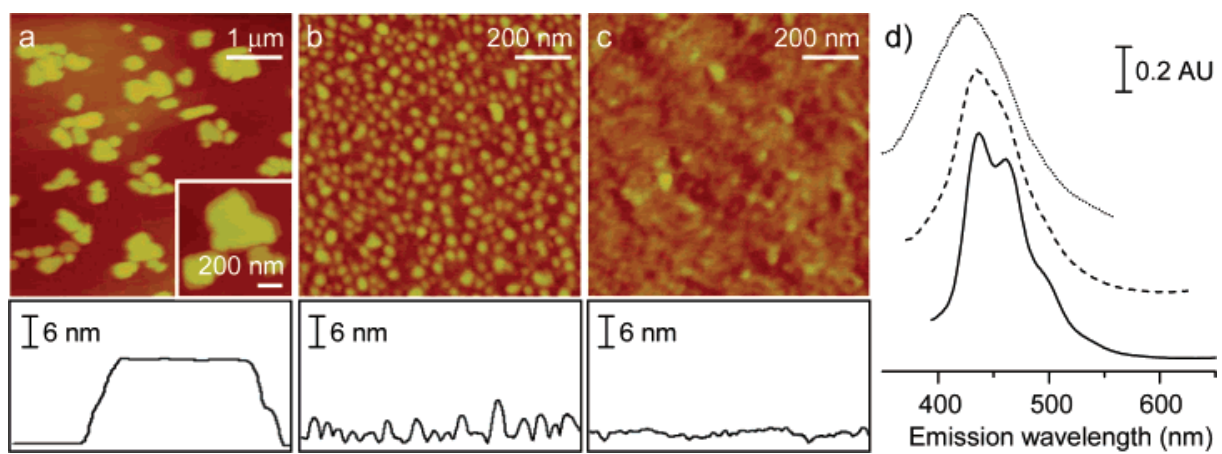

Figure 4. TM-AFM images, section profiles, and normalized PL spectra of 2-nm BTP films exhibiting features of (a) crystallinity on HOPG, (b) granules on Si-wafer, and (c) amorphism on ITO where the respective spectra are shown as solid, dashed, and dotted traces in panel (d). The vertical scales cover $40 \mathrm{~nm}$ for panel a and $15 \mathrm{~nm}$ for panels b and c. The section profiles (height mode) shown in the lower panels correspond to an arbitrary cutaway section running across the images where the trace in panel a is associated with that presented in the inset. The films were prepared by thermal evaporation, and the 2-nm film thickness was estimated by QCM during deposition. Imaging conditions were similar to those of Figure 3.

TABLE 1: Summary of the Grain Size and the PL Characteristics of the BTP Films

\begin{tabular}{lccccc}
\hline & & \multicolumn{2}{c}{ emission spectra } & & \multicolumn{2}{c}{ excitation spectra } \\
\cline { 3 - 5 } substrate & $\begin{array}{c}\text { grain size } \\
(\mathrm{nm})\end{array}$ & $\begin{array}{c}\text { peak position } \\
(\mathrm{nm})\end{array}$ & $\begin{array}{c}\text { FWHM } \\
(\mathrm{nm})\end{array}$ & $\begin{array}{c}\text { peak position } \\
(\mathrm{nm})\end{array}$ & $\begin{array}{l}\text { FWHM } \\
(\mathrm{nm})\end{array}$ \\
\hline HOPG & $437 \pm 178$ & $435,464,501(\mathrm{sh})$ & 62 & $296(\mathrm{sh}), 351,372$ \\
Si wafer & $55 \pm 13$ & 435 & 74 & $266,330(\mathrm{sh})$ & 100 \\
ITO & & 430 & 108 & 335
\end{tabular}

panels reveal flat plateaus, grainy outlines, and relatively smooth traces, suggesting that the BTP films are crystalline on graphite, granular on untreated $\mathrm{Si}$ wafers, and amorphous on ITO electrodes. Although the influence of molecular structures, substrate, and deposition conditions on film morphology is an important subject, the details will be left for future investigation because it is complicated and not the focus of this study.

PL spectra of the three morphological features are presented in Figure 4d and are summarized in Table 1. The films prepared on HOPG are highly crystalline and exhibit a significantly narrower FWHM (solid trace) yet the positions of the $\lambda_{\max }$ differ only by $5 \mathrm{~nm}$ than those of the other two morphologies. It is thus difficult to differentiate whether the red shift in $\lambda_{\max }$ is due to the difference in their emission pathways or a result of the increased domain size (namely, the extent of aromatic $\pi-\pi$ stacking). To clarify this question, excitation spectra for the three BTP films are measured and displayed in Figure 5. When $\lambda_{\mathrm{em}}$ is monitored at 435, 464, and $501 \mathrm{~nm}$ for crystalline films on HOPG, the normalized excitation spectra (solid trace) appear identical (Figure S1 in Supporting Information). Therefore, the three emission bands have the same excitation pathway rather

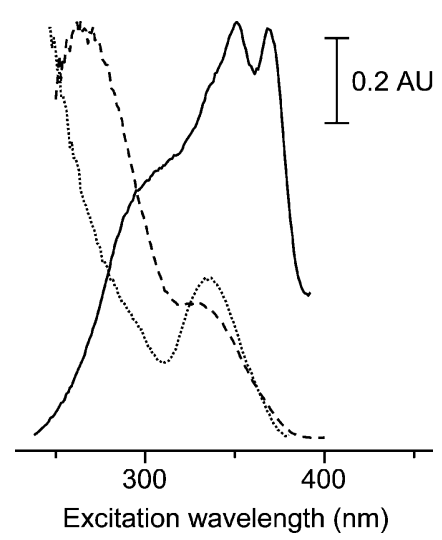

Figure 5. Normalized excitation spectra of BTP films on HOPG (solid trace, $\lambda_{\mathrm{em}}$ at $435 \mathrm{~nm}$ ), Si-wafer (dashed, $\lambda_{\mathrm{em}}$ at $435 \mathrm{~nm}$ ), and ITO (dotted, $\lambda_{\mathrm{em}}$ at $430 \mathrm{~nm}$ ). Peak positions are summarized in Table 1. than arising from different conformers as what was previously proposed. ${ }^{43}$ The excitation and emission spectra (Figure 4d) appear as approximate mirror images, suggesting that the spacing between vibrational levels for the excitation state is roughly equal to that of the ground state.

For the film morphology of $2 \mathrm{~nm}$ BTP on Si wafer (Figure 4b), TM-AFM reveals grainy aggregates with an average diameter and height of $53 \pm 13$ and $4.2 \pm 1.3 \mathrm{~nm}$, respectively. This morphological feature closely resembles that at the initial stage of BTP evaporation on a $40 \mathrm{~nm}$ NPB underlayer (Figure $3 \mathrm{c})$. Note that the emission from NPB frustrates PL studies of BTP in the device layout. Figure 5 displays the excitation spectrum (the dashed curve) of the BTP nanoaggregates on the $\mathrm{Si}$ wafer. The spectrum is distinctly different than that of crystalline structures on graphite, indicative of dissimilar excitation pathways. In fact, the spectra from the grainy aggregates on $\mathrm{Si}$ wafer and the amorphous films on ITO are relatively similar. Hence, the relative energy between rotational conformations (Figure 6) is calculated to approximate possible BTP structures in the nanoaggregates.

By B3LYP/6-31G* method in gas phase, BTP isomers of cis and trans forms are found having close potential energies with dihedral angles of 39 and $143^{\circ}$, respectively. Due to the

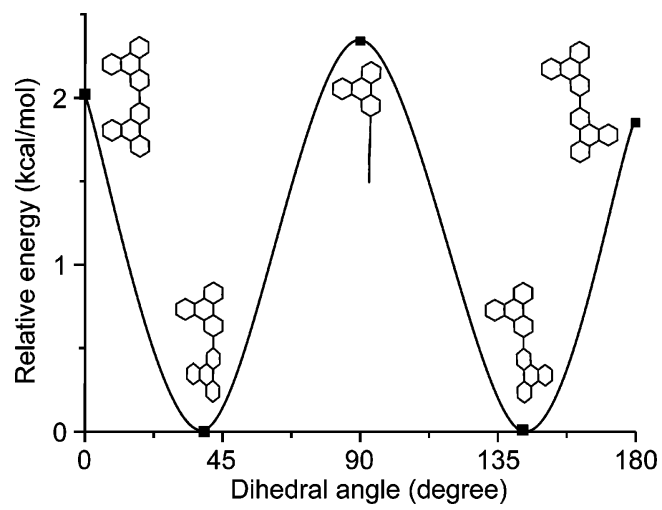

Figure 6. Calculated torsion potentials (B3LYP/6-31G* + ZPE level) of BTP as a function of dihedral angle between the triphenylenyls. 
contribution of the conjugation between TP rings, the minimum energies are at these angles rather than that of $90^{\circ}$ for minimal steric interactions. Conformations with maximum energies are found at the dihedral angles of 0 , near 90 , and $180^{\circ}$. Figure 6 shows that the planarization energy is smaller than $3 \mathrm{kcal} / \mathrm{mol}$ and below the crystal packing forces. Therefore, it is facile to rotate the triphenylenyl planes and explains the coplanar arrangement of BTP in the crystal form that is stabilized by $\pi-\pi$ stacking between the neighboring $\mathrm{J}$-aggregated aromatics. The structure of cis and trans isomers of BTP and the relative energies as a function of dihedral angles between the two triphenylenyls are provided in Supporting Information.

The closeness in $\lambda_{\max }$ between the EL spectra of the device and PL of BTP powders (Figure 2) suggest the $\pi$-conjugation system for the nanoaggregates in OLED devices is extended intermolecularly via $\pi-\pi$ stacking or intramolecularly due to a small dihedral angle. Nevertheless, BTP nanoaggregates prepared on $\mathrm{Si}$ wafer bear a very different excitation spectrum than that of BTP crystals on HOPG (Figure 5), indicating that the molecular conformations in nanoaggregates deviate from the coplanarity in crystal. To further explore the spectral features, time-dependent DFT (TD-DFT) at the B3LYP/6-31G* level was carried out to find vertical transitions and oscillator strength for BTP $\mathrm{S}_{n} \leftarrow \mathrm{S}_{0}$ at dihedral angles of $0,39,90,143$, and $180^{\circ}$. The results are tabulated in Table $\mathrm{S} 2$ of the Supporting Information. For coplanar conformations, the transitions with large oscillator strengths are at 286 and $346 \mathrm{~nm}$, similar to the 296 and $351 \mathrm{~nm}$ found in the excitation spectra of the crystalline BTP on HOPG. For those with dihedral angles of 39 and $143^{\circ}$, the transitions are at 280 and $330 \mathrm{~nm}$, close to the 266 and 330 $\mathrm{nm}$ in the spectra of aggregated BTP on Si wafer (Figure 5). Another important fact is the broadness of the EL spectra, suggesting a mixture of BTP conformations present in the thin film. Taking together the aforementioned observations, the triphenylenyls in the BTP nanoaggregates have dihedral angles ranging from around $39^{\circ}$ (and $\sim 143^{\circ}$ ) to that of coplanarity.

\section{Concluding Remarks}

BTP is an underivatized polyaromatic compound whose two halves of triphenylenyls are determined coplanar in crystal by X-ray crystallography. Absorbance spectra suggest aggregation of BTP taking place in thin films yet the EL performance shows high efficiency, indicating that self-quenching, which is common for many underivatized planar aromatics, is not the dominant pathway of energy relaxation. To correlate luminescent spectra with film morphology, TM-AFM is employed to examine the BTP layer in the device layout and reveals grainy nanoaggregates. BTP tends to aggregate on HOPG, the NPB layer, and Si wafer, attributable to a strong intermolecular $\pi-\pi$ interaction. The measurements of PL, excitation spectra, morphological images, and simulations suggest that in the OLED devices BTP molecules form nanoaggregates by adopting a range of dihedral angles that grant BTP not self-quenched even without being derivatized with steric side chains.

Acknowledgment. Financial support from the Ministry of Economics (95-EC17-A-08-S1-042) and computation facility from NCHC (National Center for High Performance Computing) are gratefully appreciated.

Supporting Information Available: Excitation spectra of BTP films on HOPG and simulation results. This material is available free of charge via the Internet at http://pubs.acs.org.

\section{References and Notes}

(1) Mi, B. X.; Gao, Z. Q.; Lee, C. S.; Kwong, H. L.; Wang, N. B.; Lee, S. T. J. Mater. Chem. 2001, 11, 2244.

(2) Seguy, I.; Destruel, P.; Bock, H. Synth. Met. 2000, 111, 15.

(3) Seguy, I.; Jolinat, P.; Destruel, P.; Farenc, J.; Mamy, R.; Bock, H.; Ip, J.; Nguyen, T. P. J. Appl. Phys. 2001, 89, 5442.

(4) Bendikov, M.; Wudl, F.; Perepichka, D. F. Chem. Rev. 2004, 104, $4891-4945$.

(5) Pisula, W.; Menon, A.; Stepputat, M.; Lieberwirth, I.; Kolb, U.; Tracz, A.; Sirringhaus, H.; Pakula, T.; Mullen, K. Adv. Mater. 2005, 17 , 684.

(6) van de Craats, A. M.; Stutzmann, N.; Bunk, O.; Nielsen, M. M.; Watson, M.; Mullen, K.; Chanzy, H. D.; Sirringhaus, H.; Friend, R. H. Adv. Mater. 2003, 15, 495.

(7) Ackermann, J.; Videlot, C.; El Kassmi, A. Thin Solid Films 2002, 403-404, 157-161.

(8) Li, J.; Osasa, T.; Hirayama, Y.; Sano, T.; Wakisaka, K.; Matsumura, M. Sol. Energy Mater. Sol. Cells 2007, 91, 745-750.

(9) Adams, D. M.; Kerimo, J.; Olson, E. J. C.; Zaban, A.; Gregg, B. A.; Barbara, P. F. J. Am. Chem. Soc. 1997, 119, 10608-10619.

(10) Hiromitsu, I.; Murakami, Y.; Ito, T. J. Appl. Phys. 2003, 94, 24342439.

(11) Cremer, J.; Bäuerle, P. Eur. J. Org. Chem. 2005, 3715-3723.

(12) Aroca, R.; Caño, T. D.; de Saja, J. A. Chem. Mater. 2003, 15, $38-45$.

(13) Tang, C. W. Appl. Phys. Lett. 1986, 48, 183-185.

(14) Han, E. M.; Do, L. M.; Niidome, Y.; Fujihira, M. Chem. Lett. 1994, 969-972.

(15) Cuppen, H. M.; Graswinckel, W. S.; Meekes, H. Cryst. Growth Des. 2004, 4, 1351-1357.

(16) Joswick, M. D.; Campbell, I. H.; Barashkov, N. N.; Ferraris, J. P. J. Appl. Phys. 1996, 80, 2883-2890.

(17) Strohriegl, P.; Grazulevicius, J. V. Adv. Mater. 2002, 14, 14391452.

(18) Wang, S.; Oldham, W. J., Jr.; Hudack, R. A., Jr.; Bazan, G. C. J. Am. Chem. Soc. 2000, 122, 5695-5709.

(19) Han, E.-M.; Do, L.-M.; Yamamoto, N.; Fujihira, M. Thin Solid Films 1996, 273, 202-208

(20) Balakrishnan, K.; Datar, A.; Naddo, T.; Huang, J.; Oitker, R.; Yen, M.; Zhao, J.; Zang, L. J. Am. Chem. Soc. 2006, 128, 7390-7398.

(21) Schouwink, P.; Schafer, A. H.; Seidel, C.; Fuchs, H. Thin Solid Films 2000, 372, 163-168.

(22) Wang, J.; Zhao, Y.; Zhang, J.; Zhang, J.; Yang, B.; Wang, Y.; Zhang, D.; You, H.; Ma, D. J. Phys. Chem. C 2007, 111, 9177-9183.

(23) Wang, J.; Zhao, Y.; Dou, C.; Sun, H.; Xu, P.; Ye, K.; Zhang, J.; Jiang, S.; Li, F.; Wang, Y. J. Phys. Chem. B 2007, 111, 5082-5089.

(24) Blochwitz, J.; Pfeiffer, M.; Hofmann, M.; Leo, K. Synth. Met. 2002 127, 169-173.

(25) Chen, J. P.; Tanabe, H.; Li, X.-C.; Thoms, T.; Okamura, Y.; Ueno, K. Synth. Met. 2003, 132, 173-176

(26) Shirota, Y. J. Mater. Chem. 2000, 10, 1-25.

(27) Kulkarni, A. P.; Gifford, A. P.; Tonzola, C. J.; Jenekhe, S. A. Appl. Phys. Lett. 2005, 86, 061106.

(28) Hancock, J. M.; Gifford, A. P.; Zhu, Y.; Lou, Y.; Jenekhe, S. A Chem. Mater. 2006, 18, 4924-4932.

(29) Tonzola, C. J.; Kulkarni, A. P.; Gifford, A. P.; Kaminsky, W.; Jenekhe, S. A. Adv. Funct. Mater. 2007, 17, 863-874.

(30) Hancock, J. M.; Gifford, A. P.; Tonzola, C. J.; Jenekhe, S. A. J. Phys. Chem. C 2007, 111, 6875-6882.

(31) Thomas, K. R. J.; Velusamy, M.; Lin, J. T.; Chuen, C. H.; Tao, Y.-T. J. Mater. Chem. 2005, 15, 4453-4459.

(32) Yeh, H.-C.; Lee, R.-H.; Chan, L.-H.; Lin, T.-Y. J.; Chen, C.-T.; Balasubramaniam, E.; Tao, Y.-T. Chem. Mater. 2001, 13, 2788-2796.

(33) Tao, S.; Peng, Z.; Zhang, X.; Wang, P.; Lee, C.-S.; Lee, S.-T. Adv. Funct. Mater. 2005, 15, 1716-1721.

(34) Robinson, M. R.; Wang, S.; Bazan, G. C.; Cao, Y. Adv. Mater 2000, 12, 1701-1704.

(35) Wu, C. C.; Lin, Y. T.; Chiang, H. H.; Cho, T. Y.; Chen, C. W.; Wong, K. T.; Liao, Y. L.; Lee, G. H.; Peng, S. M. Appl. Phys. Lett. 2002 $81,577-579$.

(36) Steuber, F.; Staudigel, J.; Stossel, M.; Simmerer, J.; Winnacker, A.; Spreitzer, H.; Weissortel, F.; Salbeck, J. Adv. Mater. 2000, 12, 130133.

(37) Salbeck, J.; Yu, N.; Bauer, J.; Weissortel, F.; Bestgen, H. Synth. Met. 1997, 91, 209-215.

(38) Johansson, N.; dos Santos, D. A.; Guo, S.; Cornil, J.; Fahlman, M.; Salbeck, J.; Schenk, H.; Arwin, H.; Bredas, J. L.; Salanek, W. R. J. Chem. Phys. 1997, 107, 2542-2549.

(39) Benmansour, H.; Shioya, T.; Sato, Y.; Bazan, G. C. Adv. Funct. Mater. 2003, 13, 883-886. 
(40) He, Q.; Lin, H.; Weng, Y.; Zhang, B.; Wang, Z.; Lei, G.; Wang, L.; Qiu, Y.; Bai, F. Adv. Funct. Mater. 2006, 16, 1343-1348.

(41) Ostrowski, J. C.; Hudack, R. A.; Robinson, M. R.; Wang, S.; Bazan, G. C. Chem.-Eur. J. 2001, 7, 4500-4511.

(42) Zhou, Q.; Qin, A.; He, Q.; Lei, G.; Wang, L.; Qiu, Y.; Ye, C.; Teng, F.; Bai, F. J. Lumin. 2007, 122, 674-677.

(43) Shih, H.-T.; Lin, C.-H.; Shih, H.-H.; Cheng, C.-H. Adv. Mater. 2002, 14, 1409-1412.

(44) García, R.; Pérez, R. Surf. Sci. Rep. 2002, 47, 197-301.

(45) Giessibl, F. J. Rev. Mod. Phys. 2003, 75, 949-983.

(46) Knoll, A.; Magerle, R.; Krausch, G. Macromolecules 2001, 34, $4159-4165$

(47) Shih, H.-T.; Shih, H.-H.; Cheng, C.-H. Org. Lett. 2001, 3, 811814.
(48) Becke, A. D. Phys. Rev. A 1988, 38, 3098-3100.

(49) Lee, C.; Yang, W.; Parr, R. G. Phys. Rev. B 1988, 37, 785-789.

(50) Pavia, D. L.; Lampman, G. M.; Kriz, G. S. Ultraviolet Spectroscopy. In Introduction to Spectroscopy: A Guide for Students of Organic Chemistry; Thomson Learning: Stamford, CT, 2001; pp 353-386.

(51) Tirapattur, S.; Belletete, M.; Drolet, N.; Leclerc, M.; Durocher, G. Macromolecules 2002, 35, 8889-8895.

(52) Antolini, L.; Tedesco, E.; Barbarella, G.; Favaretto, L.; Sotgiu, G.; Zambianchi, M.; Casarini, D.; Gigli, G.; Cingolani, R. J. Am. Chem. Soc. 2000, 122, 9006-9013.

(53) Yang, A.; Kuroda, M.; Shiraishi, Y.; Kobayashi, T. J. Chem. Phys. 1998, 109, 8442-8450. 